\title{
对称可区分的三维人体模型语义分割
}

\author{
罗闪, 张栖桐, 冯结青* \\ (浙江大学 CAD\&CG 国家重点实验室 杭州 310058) \\ (jqfeng@cad.zju.edu.cn)
}

\begin{abstract}
摘 要: 针对三维人体模型语义获取和对称区分的难题，提出一种自动的、左右可区分的三维人体模型语义分割方 法. 对于输人的人体模型，首先采用模板嵌人的方法提取其左右可区分的、具有语义信息的运动骨架; 然后基于运动 骨架确定人体模型的分割块数量, 并提取各个分割块对应的关键点; 再以关键点为边界约束计算模型的调和场, 获 取分割线的候选集; 最后以关键点为聚类中心对模型进行谱聚类, 利用聚类结果引导分割线的篮选，确定其中准确 的分割线. 该方法使骨架的语义信息可直接传递至三维人体模型的分割块, 且借助骨架的结构可以控制分割块的数 量. 在 SCAPE 数据库、MPI-FAUST 数据库和普林斯顿分割数据集上的结果表明, 对不同形状和姿态的三维人体模型, 该方法均能自动、鲁棒地实现对称可区分的语义分割.
\end{abstract}

关键词: 三维人体模型; 对称可区分; 语义分割; 调和场; 谱聚类

中图法分类号: TP391.41 DOI: 10.3724/SP.J.1089.2021.18638

\section{Symmetry-Aware Semantic Segmentation of a 3D Human Body Model}

\author{
Luo Shan, Zhang Qitong, and Feng Jieqing* \\ (State Key Laboratory of CAD\&CG, Zhejiang University, Hangzhou 310058)
}

\begin{abstract}
An automatic segmentation method for a 3D human model is proposed for the symmetry-aware semantic segmentation. Firstly, a symmetry-aware kinematic skeleton is extracted from the input human body model via template skeleton embedding. Secondly, the number of the segments is determined by the kinematic skeleton; some key points corresponding to the segments are extracted meanwhile. Thirdly, while taking the key points as the boundary constraints, the harmonic fields are constructed to obtain a set of isolines as the potential cutting boundaries. Finally, the key points are taken as the clustering centers to conduct a spectral clustering operation, which guides the selections of the cutting boundaries. In this way, the semantic information in the kinematic skeleton can be directly transferred to the segments, and the number of segments can be controlled by the structure of the kinematic skeleton. Results on the SCAPE dataset, the MPI-FAUST dataset and the Princeton Segmentation Benchmark show that the proposed method can automatically achieve symmetry-aware semantic segmentation, and is robust to the shape and the pose of the human body model.
\end{abstract}

Key words: 3D human body model; symmetry-aware; semantic segmentation; harmonic field; spectral clustering

收稿日期：2020-09-04; 修回日期：2020-11-16. 基金项目：国家自然科学基金(61732015, 61472349); 浙江省重点研发计划 (2018C01090). 罗闪(1993-), 女, 博士研究生, CCF 会员, 主要研究方向为三维模型的数字几何处理; 张栖桐(1996一), 女, 博士研 究生, 主要研究方向为计算机视觉; 冯结青(1970一), 男, 博士, 教授, 博士生导师, CCF 杰出会员, 论文通讯作者, 主要研究方向为 计算机图形学、计算机视觉、光热太阳能仿真等. 
三维模型分割是数字几何处理中的一个基本 问题, 在计算机图形学中具有广泛的应用, 如模型 一致参数化、参数化建模、网格变形、模型检索等. 三维人体模型作为一种特殊且重要的三维模型, 其分割块的语义信息在后续的应用中具有重要意 义. 一般的三维模型分割方法虽然能得到模型的 分割结果，但通常无法获取模型分割块的语义信 息. 基于监督学习的分割方法 ${ }^{[1-3]}$ 以人工分割的模 型进行训练, 测试得到的模型分割块数量和语义 信息均与训练模型保持一致. 但此类方法需要手 工标记训练数据, 标记工作乏味且耗时. 因此, 如 何直接对三维模型进行语义分割是一个具有挑战 性的问题.

骨架作为模型的一种抽象表示，其语义信息 可以传递给模型. 目前, 已有方法 ${ }^{[4]}$ 可以从三维人 体模型中提取带有语义信息的运动骨架. 相比基 于监督学习的分割方法, 通过骨架获取分割块的 语义信息更加简单方便，无需大量手工标记的训 练数据. 这项工作提示我们: 是否可以结合具有语 义信息的运动骨架和传统三维模型分割方法, 直 接实现三维人体模型的语义分割?

根据分割策略的不同，传统的三维模型分割 方法可以分为 2 类 $^{[5]}$ : 基于区域相似性检测的方法 和基于分割边界检测的方法.

基于区域相似性检测的方法有各种不同的形

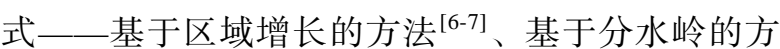
法 $^{[8-9]}$ 、基于层次聚类的方法 ${ }^{[10-11]}$ 、基于迭代聚类 的方法 ${ }^{[12-13]}$ 和基于谱聚类的方法 ${ }^{[5,14]}$ 等. 此类方法 通过对模型几何特征或拓扑特征进行分析, 以其 作为约束项或聚类的判断准则将模型划分成多个 特征相似的区域，完成三维模型的分割. 对于特征 差异明显的区域，基于区域相似性检测的分割方 法能得到比较准确的分割线. 但此类方法通常不 能自动确定分割块的数量, 容易得到欠分割或过 分割的结果. 虽然部分方法通过人工指定种子点 或指定分割块的数量避免过分割, 但由于此类方 法依赖模型的特征，部分特征区分度不大的区域 仍无法得到满意的分割结果.

基于分割边界检测的方法以人眼视觉理论的 最小准则为分割标准, 即分割边界往往位于模型 的凹区域 ${ }^{[15]}$. 一些方法通过分析模型表面的曲率 信息来获取模型的分割边界 ${ }^{[16-17]}$. Tong 等 ${ }^{[18]}$ 通过 定义模型边的权重, 采用最小割的方式对模型进 行分割. 部分方法 ${ }^{[19-23]}$ 则通过计算模型的调和场,
选取合适的等值线作为模型的分割边界. 调和场 的边界可以通过手工交互的方式确定 ${ }^{[19-21]}$, 也可 以通过算法自动确定 ${ }^{[22-23]}$. 此类方法根据模型的 显著性特征识别分割边界, 分割边界之间自动形 成分割块. 但此类方法也无法自动确定分割块的 数量, 识别模型在语义上待分割的区域. 手工交互 的方式虽然能根据人类需求对模型进行分割，但通 常需要多次交互来完成模型的语义分割, 且模型分 割结果的质量往往取决于手工交互的初始位置.

针对三维人体模型，本文提出了一种自动的、 左右可区分的语义分割方法. 借助模型运动骨架 的语义信息, 本文方法可以自动地识别模型需要 分割的区域，生成对应调和场的边界约束，避免了 手工交互. 同时，本文方法采用不同策略对调和场 分割线进行篮选，并以谱聚类的分割结果引导模 型特征差异明显区域的分割线篮选，获取其中准 确的分割线.

\section{1 本文方法概述}

本文提出的自动的、对称可区分的三维人体模 型语义分割方法的框架如图 1 所示. 输人为一个三 维人体模型和一个模板骨架. 其中, 模板骨架包含 关节点的语义信息和模型分割后的骨骼标签信息. 多块骨骼可以对应同一个分割块，部分骨骼也可 以不对应任何分割块, 仅起连接作用. 输出为三维 人体模型对称可区分的语义分割结果. 图 2 给出了 一组模板骨架分割标签和模型分割结果的对应关 系. 其中, 无对应分割块的骨骼用浅灰色表示(如 髋骨骼和肩骨骼)，分割标签相同的骨骼用相同的 颜色表示.

本文方法有 5 个步骤.

Step1. 模型的骨架提取.

Step2. 模型的关键点提取.

Step3. 以关键点为聚类中心对模型进行谱聚类.

Step4. 以关键点为边界约束计算模型的调和场, 获取分割线候选集

Step5. 利用谱聚类的结果引导分割线的篮选, 获 取其中准确的分割线.

其中, Step1 中模型的骨架采用文献[4]方法进 行提取, 得到与模板骨架结构和语义一致的运动 骨架; Step3 和 Step4 均基于关键点的提取，二者没 有先后顺序. 在对左右可区分的骨架进行展示时, 骨架左侧的骨骼用蓝色表示, 其他骨骼用黄色表 示, 如图 1 所示. 


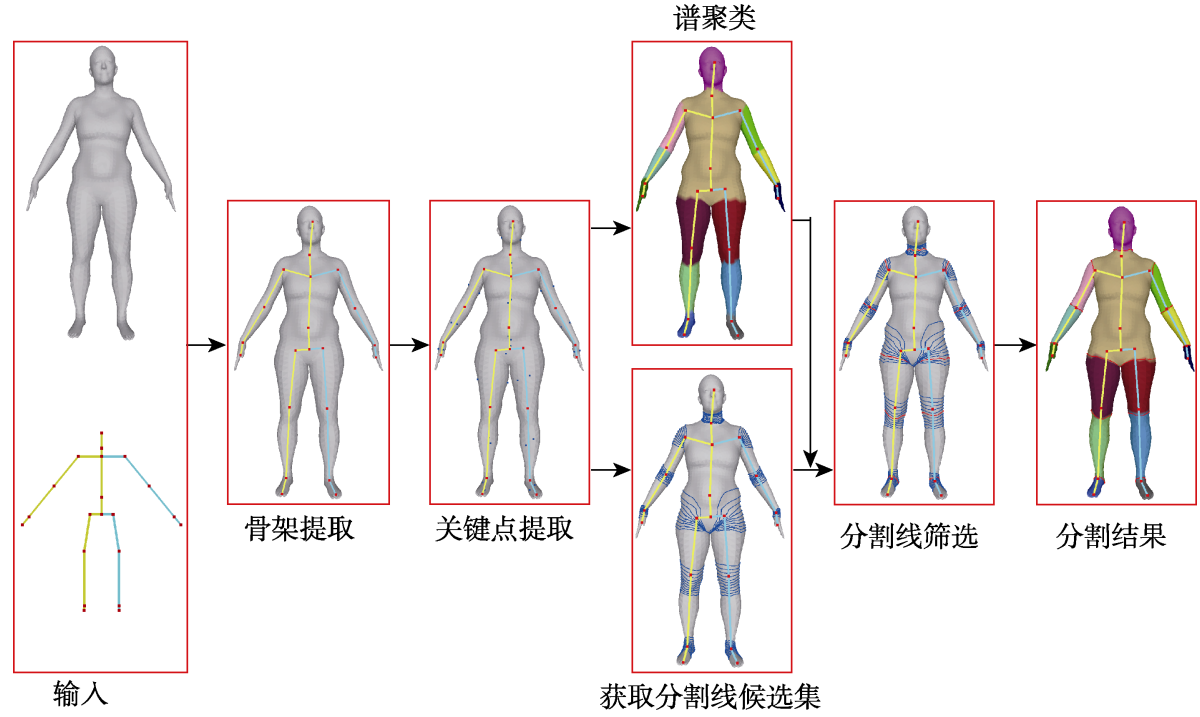

图 1 本文方法框架

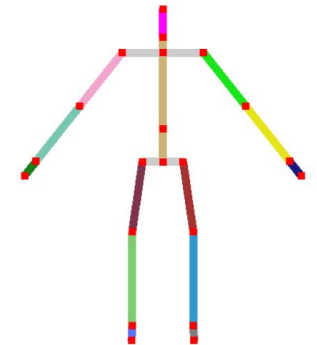

a. 模板骨架的分割标签

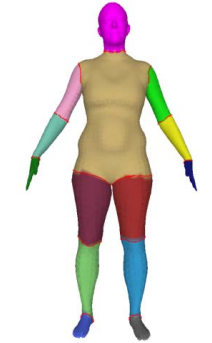

b. 模型分割结果
图 2 模板骨架分割标签与其对应分割结果

\section{2 关键点提取}

骨架的分割标签建立了骨骼和模型分割块之 间对应关系. 对于每块骨骼，提取若干个属于骨骼 对应分割块的模型顶点, 称为关键点. 这些关键点 在后续步骤中有重要作用: (1) 以关键点为聚类中 心对模型顶点进行谱聚类; (2) 以关键点为边界约 束计算模型的调和场.

对于谱聚类，每个分割块对应的关键点越多， 越能准确表示分割块的特征属性, 得到更加准确 的聚类结果. 但关键点越多, 在求解模型的调和场 时, 对调和场的边界约束则越多, 模型结构对调和 场的影响就越小，使调和场生成的分割边界更依 赖于边界而非模型结构. 综合上述考虑, 本文针对 每块骨骼, 选取了 2 个关键点. 因此, 对于每个分 割块，至少有 2 个对应的关键点，多块骨骼对应的 分割块则有多个关键点

对于有对应分割块的骨骨，如图 3a 中红色骨 骼所示，与骨骼距离越近的顶点属于骨骼对应的 分割块的概率越大. 获取模型关键点的过程如下:
以骨骼的中点为代表点，获取其横截平面; 在其截 平面上以骨骼中心径向均匀采样 $N$ 个方向; 选取 径向距离最短的采样方向为关键点的采样方向; 与关键点采样方向上的交点距离最近的模型顶点 即为该骨骼及其分割块对应的关键点. 采样方向 与模型的交点和部分采样方向如图 3a 所示.

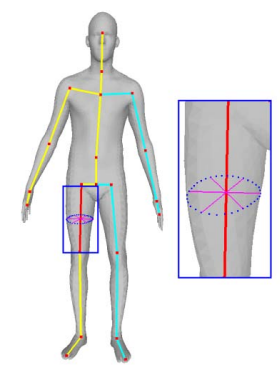

a. 有对应分割块的骨骼

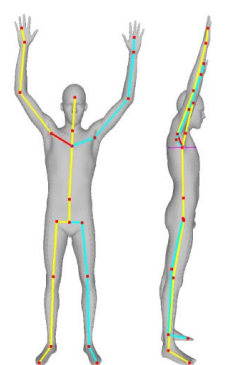

b. 无对应分割块的骨骼
图 3 骨骼的采样方向

对于无对应分割块的骨骼, 如图 3b 中红色骨 骼所示, 本文也进行了关键点提取. 这些关键点提 取的目的是为调和场的计算提供边界约束. 无对 应分割块的骨骼一般出现在结构复杂的多分支关 节点处, 为降低关节点的误差对关键点提取的影 响, 本文以更鲁棒的多分支关节点为代表点, 以与 其相邻的其他关节点拟合的平面的法向量为关键 点的采样方向, 如图 3b 所示, 直接确定其对应的 2 个关键点.

\section{3 谱聚类}

模型的关键点由骨架直接提取, 因此关键点 的分割标签与骨架上各骨骼保持一致, 使每一个 
分割块均有与其对应的关键点. 本文直接以关键 点为聚类中心, 采用与文献[14]相似的谱特征对模 型顶点进行聚类分析.

模型的谱特征与模型的 Laplacian 矩阵相关. 在本文中, 模型的 Laplacian 矩阵的定义为

$$
L_{i j}= \begin{cases}w_{i j}, & i \neq j, \quad(i, j) \in E \\ -\sum_{k} w_{i k}, & i=j, k \in N(i) . \\ 0, & \text { 其他 }\end{cases}
$$

其中， $E$ 表示模型的边集合; $N(i)$ 表示模型顶点 $v_{i}$ 的邻接顶点的集合; $w_{i j}$ 为顶点 $v_{i}$ 和顶点 $v_{j}$ 之间 的权重.

为了使谱聚类的结果更加符合人们在模型的 凹区域进行分割的理论，本文在定义模型顶点间 的权重时，同时考虑了模型边长和顶点间法向量 的差异. 顶点间法向量差异越大，说明该区域模型 的凹凸性变化越显著. 模型顶点间的权重 $w_{i j}$ 的具 体定义为

$$
w_{i j}=\exp \left(-\left(\frac{1}{1+\beta} \frac{\left\|\boldsymbol{p}_{i}-\boldsymbol{p}_{j}\right\|}{\bar{e}}+\frac{\beta}{1+\beta} \frac{\left\|\boldsymbol{n}_{i}-\boldsymbol{n}_{j}\right\|}{\bar{n}}\right)\right) .
$$

其中, $\boldsymbol{p}_{i}$ 和 $\boldsymbol{p}_{j}$ 分别表示顶点 $v_{i}$ 和 $v_{j}$ 的坐标; $\boldsymbol{n}_{i}$ 和 $\boldsymbol{n}_{j}$ 分别表示顶点 $v_{i}$ 和 $v_{j}$ 的法向量; $\bar{e}$ 和 $\bar{n}$ 分别表 示模型边长与模型顶点间法向量差异的均值, 即 分别为 $\left\|\boldsymbol{p}_{i}-\boldsymbol{p}_{j}\right\|$ 项和 $\left\|\boldsymbol{n}_{i}-\boldsymbol{n}_{j}\right\|$ 项的均值.

在获取模型的 Laplacian 矩阵后, 计算其前 $N_{1}$ 个最小非零特征值对应的特征向量 $\left[\boldsymbol{V}_{1}, \boldsymbol{V}_{2}, \cdots, \boldsymbol{V}_{N_{1}}\right]$. 模型顶点 $v_{i}$ 的谱特征 $\boldsymbol{f}_{i}=\left(V_{1, i}, V_{2, i}, \cdots, V_{N_{1}, i}\right)$. 以关键 点为聚类中心，利用特征相似性对模型顶点进行 聚类，有相同分割标签的关键点为同一类. 在实验 中, 取 $\beta=50, N_{1}=20$.

图 4 展示了模型以关键点为聚类中心的聚类结 果. 对于特征明显的区域，如弯曲的肘关节区域， 谱聚类能得到比较好的分割结果; 但部分区域由于 特征相似或受关键点位置的影响, 谱聚类不能得到

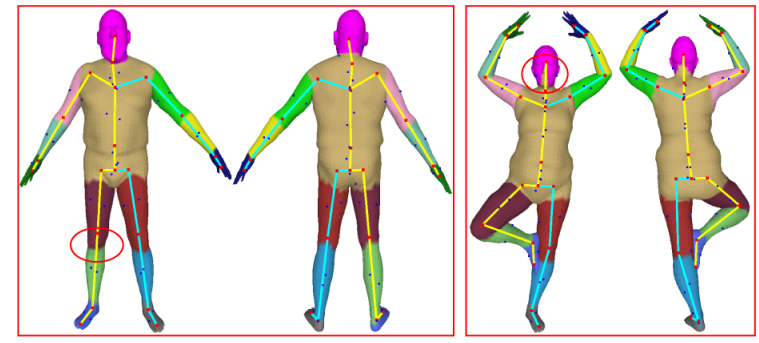

图 4 以关键点为聚类中心的聚类结果
理想的语义分割结果, 如图 4 中椭圆圈出区域所示.

\section{4 分割线获取}

分割线的获取主要有 2 个步骤: (1) 确定调和 场的边界条件；(2) 计算模型网格上的调和场，提 取若干等值线构成分割线候选集.

\section{1 调和场边界条件的确定}

骨架上每块骨骼均有与其对应的分割标签, 当相邻的 2 块骨骼的分割标签不一致时, 说明该区 域存在一条分割线. 该分割线对应的调和场的边 界条件定义为

$$
\left\{\begin{array}{l}
u_{i}=0, v_{i} \in S_{F} \\
u_{i}=1, v_{i} \in S_{B}
\end{array} .\right.
$$

其中, $S_{F}$ 和 $S_{B}$ 分别表示相邻骨骼对应的关键点的 集合; $u_{i}$ 表示关键点的边界值.

\section{2 分割线候选集的提取}

计算模型网格上的调和场通常通过求解如下 泊松方程： $\Delta \boldsymbol{\Phi}=\mathbf{0}$, 其中 $\Delta$ 表示模型表面的 Laplace-Beltrami 算子. 对于有边界值的情况, 上 述泊松方程可表示成矩阵形式: $\boldsymbol{A \Phi}=\boldsymbol{b}$, 其中,

$$
A=\left[\begin{array}{l}
L \\
P
\end{array}\right], \quad b=\left[\begin{array}{l}
0 \\
B
\end{array}\right] .
$$

矩阵 $\boldsymbol{L}$ 表示模型的 Laplacian 矩阵, 其定义详见第 3 节, 矩阵 $\boldsymbol{P}$ 和向量 $\boldsymbol{B}$ 分别表示边界点的位置及其 对应的边界值。

求解上述泊松方程, 得到模型顶点的一组标 量值. 然后在模型表面选取若干等值线，构成该区 域的分割线候选集.

由于分割线位于相邻的 2 块骨骼之间，本文通 过骨架的长度对最优分割线的位置进行了预估, 自适应地选取等值线的取值区间. 若 $S_{F}$ 和 $S_{B}$ 中关 键点对应的骨骼长度分别为 $d_{1}$ 和 $d_{2}$, 其最优分割 线的取值可能为 $\frac{d_{1}}{d_{1}+d_{2}}$. 因此, 本文将等值线的 取值区间定义为 $\left[\frac{d_{1}}{d_{1}+d_{2}}-\lambda, \frac{d_{1}}{d_{1}+d_{2}}+\lambda\right]$. 其中, $\lambda$ 表示预估误差值, 定义为

$$
\lambda=\min \left(\frac{d_{1}}{d_{1}+d_{2}}, 0.25,1-\frac{d_{1}}{d_{1}+d_{2}}\right) .
$$

在该取值区间内均匀取 $N_{2}$ 个值, 得到的 $N_{2}$ 条等 值线即构成的该分割区域的分割线候选集. 在实 验中, $N_{2}=11$. 


\section{5 分割线篮选}

在获取分割线候选集后, 需要从中篮选最优 的分割线, 得到最终的分割结果. 由于人眼视觉理 论往往趋于在模型的凹区域对模型进行分割，以 往的方法常常使用分割线的长度、分割线的凹形以 及分割线的中心性等对分割线进行筷选. 但随着 人体姿态的变化, 人体模型部分区域的凹凸性也 会发生变化, 分割线的属性也会随之发生变化, 使 上述節选条件不够鲁棒。

对于人体模型的凹区域，分割线的位置可以 通过曲率等特征确定; 但对于模型相对平坦的区 域, 由于特征的缺失导致分割线难以确定, 如伸直 的胳膊在肘关节处的分割线. 因此，采用同一种篮 选方式处理上述 2 个区域难以达到理想的分割结 果. 本文提出了一种自适应的分割线篮选方法, 对 模型凹区域和模型其他区域采用不同的方式对分 割线进行笁选.

首先，通过模型的最小主曲率将模型分成分 割特征明显的区域和分割特征不明显的区域. 对 于一个分割线候选集，若大多数分割线上点的最 小曲率值小于凹区域阈值，则该区域为分割特征 明显的区域; 否则，该区域为分割特征不明显的区 域. 凹区域阈值定义为使模型 $1 / 10$ 的顶点的曲率 小于该國值的值.

对于分割特征明显的区域，模型顶点的曲率 值差异比较明显, 谱聚类的分割结果也比较准确. 因此, 除曲率外, 还可以采用谱聚类的分割结果引 导分割线的篮选. 对于该区域，从以下 3 个方面对 分割线进行篮选.

(1) 分割线上点到 2 个分割块的谱特征差异 $F_{I_{i}}=\sum_{j \in P_{i}} l_{j}\left\|\min _{k \in S_{F}}\left(\left\|\boldsymbol{f}_{j}-\boldsymbol{f}_{k}\right\|\right)-\min _{k \in S_{B}}\left(\left\|\boldsymbol{f}_{j}-\boldsymbol{f}_{k}\right\|\right)\right\| / \sum_{j \in P_{i}} l_{j}$. 其中, $I_{i}$ 表示每条分割线; $P_{i}$ 表示组成该分割线的 点集; $l_{j}$ 表示该点在分割线上基于长度的权重值.

(2) 分割线上点的平均曲率值, 即

$$
C_{I_{i}}=\sum_{j \in P_{i}} l_{j}\left\|c_{j}\right\| / \sum_{j \in P_{i}} l_{j} .
$$

其中, $c_{j}$ 表示分割线上点的曲率值.

(3) 分割线上点的最大曲率差, 即

$$
D_{I_{i}}=\max _{j \in P_{i}} c_{j}-\min _{j \in P_{i}} c_{j} .
$$

综合考虑上述 3 个因素, 对于分割特征明显的 区域，分割线的度量值计算公式为

$$
S_{I_{i}}=\exp \left(-w_{1} F_{I_{i}}\right) \times \exp \left(C_{I_{i}}\right) \times \exp \left(D_{I_{i}}\right) .
$$

其中， $w_{1}$ 为使特征差异和曲率值保持在同一个数 量级的系数, 取值为 $w_{1}=\frac{\max _{i} C_{I_{i}}+\min _{i} C_{I_{i}}}{\max _{i} F_{I_{i}}+\min _{i} F_{I_{i}}}$. 度量 值最高的分割线即为最优分割线.

对于分割特征不明显的区域，由于特征的缺 失, 谱聚类和凹区域检测在该区域均无法获得准 确的分割结果. 本文对该区域进行分割线篮选时, 考虑骨架的关节点和分割线的中心性 2 个因素.

(1) 分割线的中心点与模型对应关节点之间 的距离为

$$
E_{I_{i}}=\left\|\left(\sum_{j \in P_{i}} l_{j} v_{j} / \sum_{j \in P_{i}} l_{j}\right)-J_{p}\right\| .
$$

其中, $J_{p}$ 表示该分割线对应的关节点的坐标.

(2) 分割线的中心性为

$$
M_{I_{i}}=\frac{\left\|i-N_{2} / 2\right\|}{N_{2} / 2} .
$$

其中, $N_{2}$ 表示分割线候选集中分割线的数量. 在 选取等值线的取值区间时，本文采用了自适应的 方式，故越靠近中心的分割线是最优分割线的可 能性越大.

综合考虑上述 2 个因素，分割线度量值的计算 方式为 $S_{I_{i}}=\exp \left(-w_{2} \overline{E_{I_{i}}}\right) \times \exp \left(-M_{I_{i}}\right)$. 其中, $\overline{E_{I_{i}}}=$ $\frac{E_{I_{i}}-\min _{i} E_{I_{i}}}{\max _{i} E_{I_{i}}-\min _{i} E_{I_{i}}}$ 为 $E_{I_{i}}$ 归一化的结果; $w_{2}$ 为分割 线中心点与关节点距离的权重值. 度量值最高的 分割线即为最优分割线. 在实验中, $w_{2}=2$.

图 5 展示了分割线篮选的结果, 其中红色的分 割线即为篮选后的最优分割线. 本文方法能自适 应地选取篮选方式, 使模型的分割特征明显的区 域和分割特征不明显的区域均能获取正确的分割 线. 篮选出最优分割线后, 本文通过 Snake 模型 ${ }^{[24]}$ 对分割线进行平滑, 得到最终的分割结果.

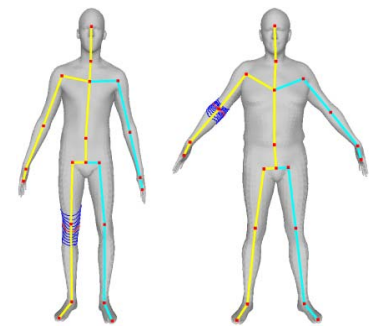

a. 分割特征不明显的区域

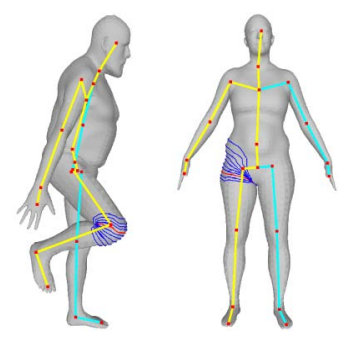

b. 分割特征明显的区域
图 5 分割线的篮选结果 


\section{6 实验结果和分析}

本文在 3 个数据集上进行了测试: (1) SCAPE 数据库 ${ }^{[25]}$, 包含同一个人的 71 个不同姿态的模型; (2) MPI-FAUST 数据库 ${ }^{[26]}$, 包含 10 个人各 10 个姿 态, 共 100 个模型; (3) 普林斯顿分割数据集 ${ }^{[27]}$, 包含 20 个人体模型.

针对单个三维人体模型，本文方法能自动地 生成其对称可区分的语义分割结果，如图 6 所示.
本文提出的人体模型分割方法能正确生成模型的 左右可区分的语义分割结果, 且对人体模型的形 状和姿态比较鲁棒. 图 6 中分割线即为模型的分割 边界. 由于分割线可以通过三角形内部, 模型在绘 制时采用对模型顶点进行着色, 因此结果展示中 分割线通过的三角面片会呈现一个渐变的颜色.

本文方法实现了分割块数量可控的语义分割, 通过修改输人模板骨架的分割标签可得到对应的 语义分割结果, 如图 7 所示.
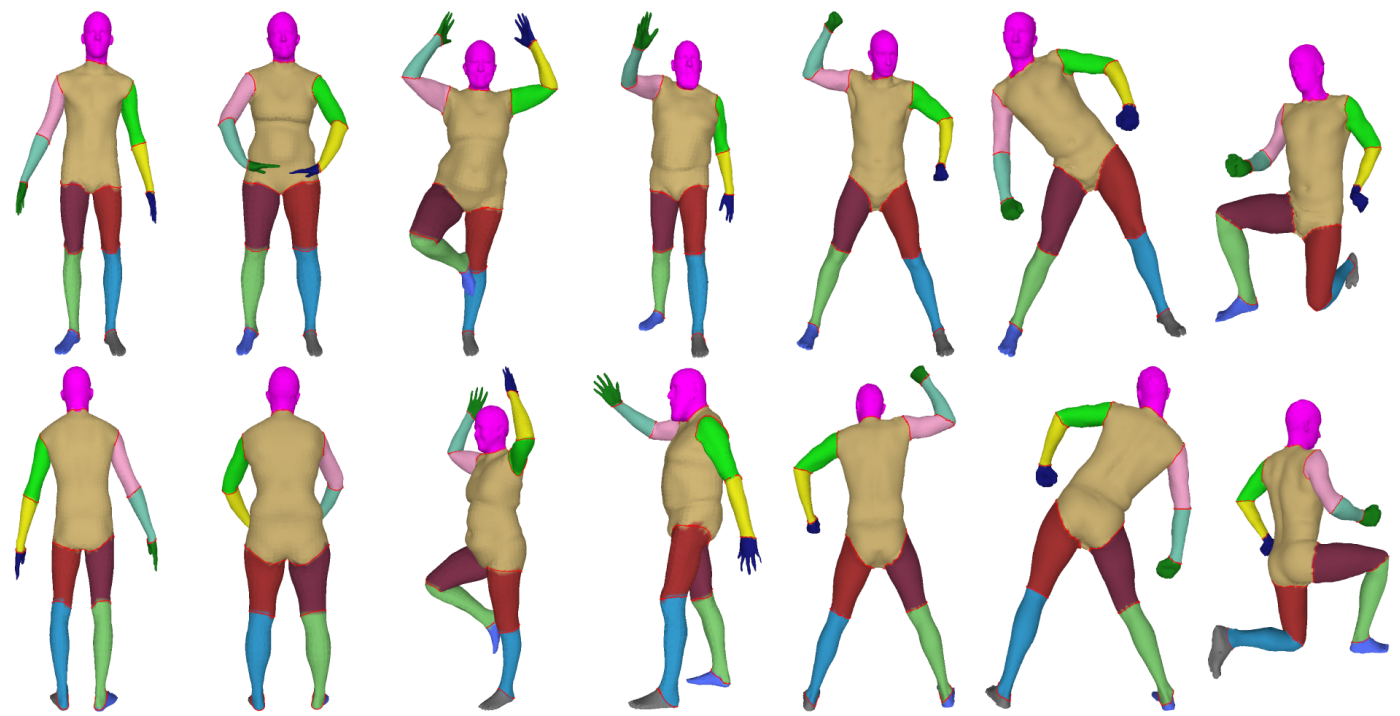

图 6 本文方法的不同视角下的语义分割结果

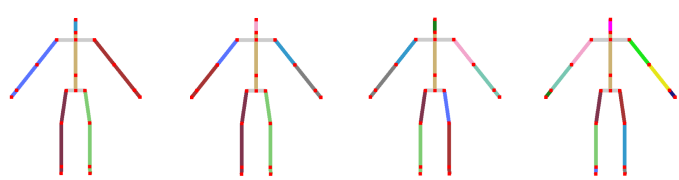

a. 分割标签

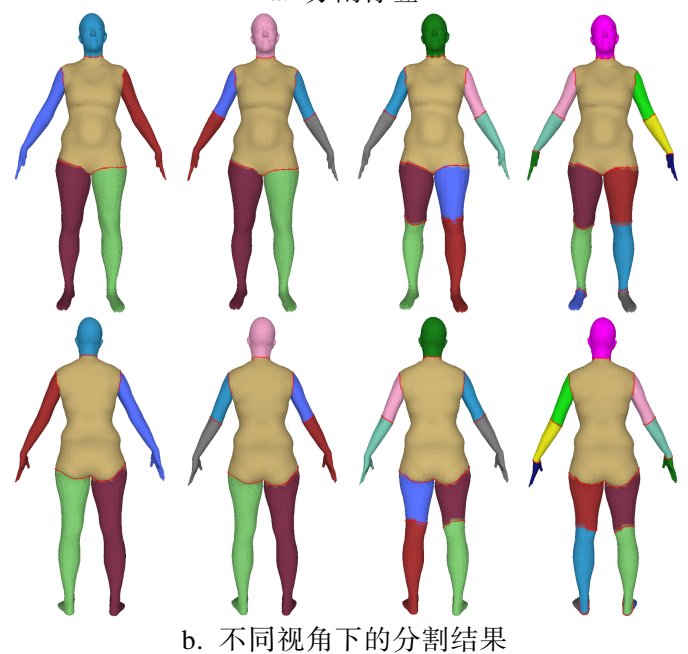

图 7 不同的分割标签对应的分割结果

本文结合了谱聚类和模型调和场的分割方法, 同时，在篮选分割线时采用了自适应的方法，对分 割特征明显的区域和分割特征不明显的区域采取
了不同的分割线篮选方式. 图 8 展示了单独采用谱 聚类和使用单一分割线篮选条件的分割结果与本 文方法分割结果的对比. 其中, 第 1 种分割线篮 选条件为分割特征明显区域的分割线篮选方法; 第 2 种分割线篮选条件为分割特征不明显区域的
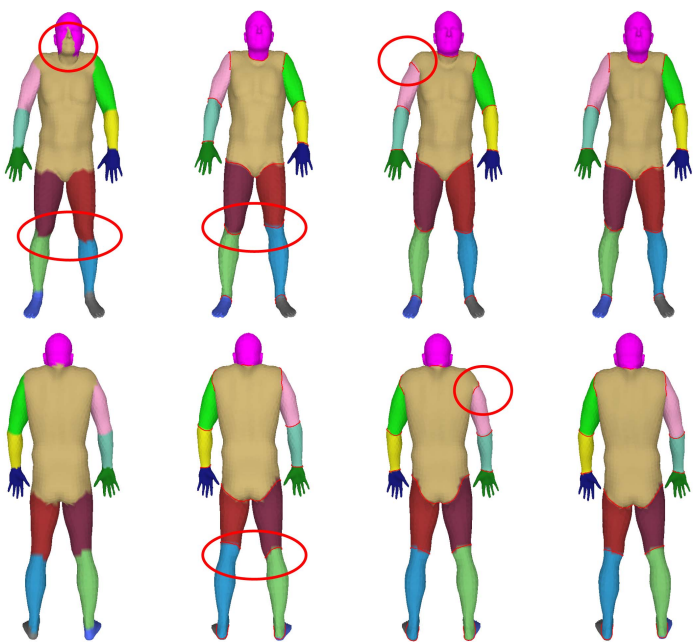

a. 谱聚类

b. 采用第 1 种分 c. 采用第 2 种分 $d$. 本文方法 割线篮选方法

图 8 本文方法与部分步骤的结果对比 
分割线篮选方法. 从图 8 可以看出, 本文方法得到 的模型分割结果明显优于部分步骤得到的结果.

图 9 给出了本文方法和一些三维模型分割方 法的结果对比，包括 2 种基于区域相似性检测的方 法 ${ }^{[10,28]}$ 和一种基于分割边界检测的方法 ${ }^{[21]}$. 同时, 图 9 也给出了本文方法与文献[1]中提供的人工分
割结果的对比. 从图 9 可以看出, 基于区域相似性 检测的方法无法确定分割块的数量, 单纯的特征 聚类无法得到语义分割的结果. 文献[21]提出一种 手工交互获取分割线的方法, 用户只需要在需分 割区域单击鼠标即可获得分割结果. 每一次交互 可以确定一条分割线, 图 9 中展示的结果是通过人
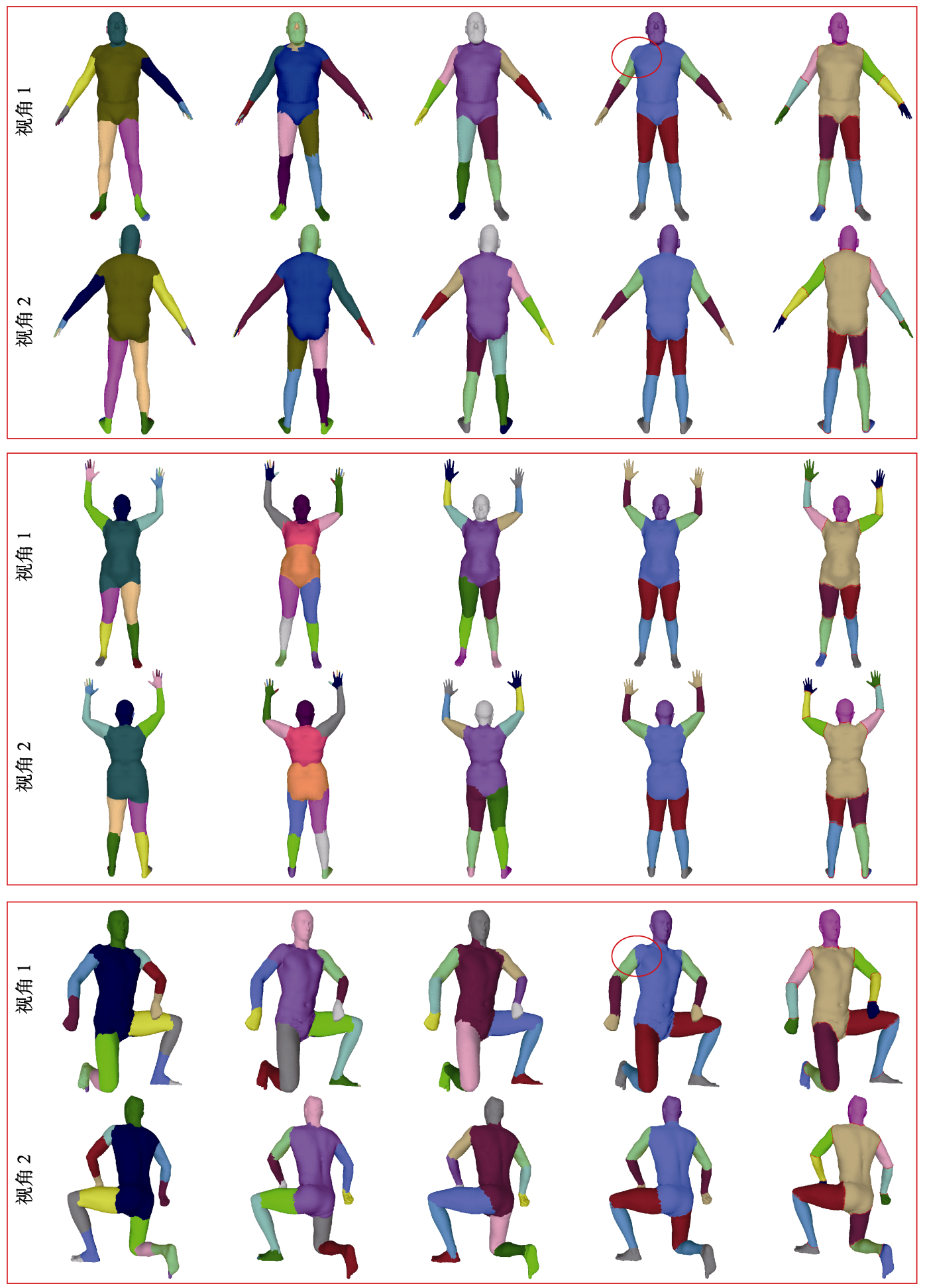

a. 文献[28]

b. 文献[10]

c. 文献[21]

d. 人工分割 ${ }^{[1]}$

e. 本文

图 9 不同分割方法的可视化结果 1 
工交互 13 次得到的分割结果. 此外，文献[21]的分 割质量严重依赖交互位置, 如图 10 所示. 本文方 法借助骨架, 自动确定分割块数量和分割线位置, 得到了左右可区分的语义分割结果. 同时，本文方 法得到的部分结果在肩部的分割线视觉上甚至优 于文献[1]中人工分割的结果，如图 9 中椭圆圈出 区域所示.

图 11 展示了本文方法、基于调和场的自动分
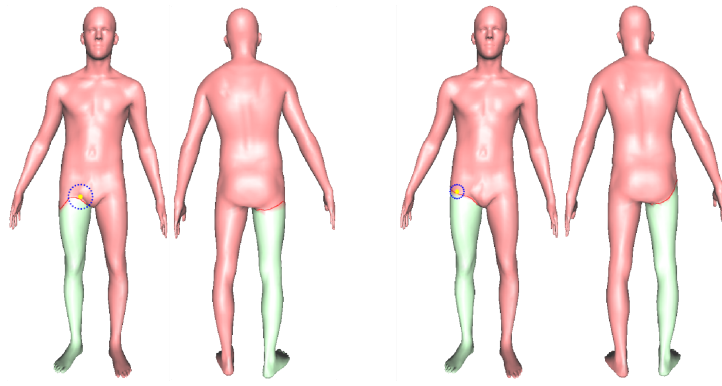

图 10 手工交互位置对文献[21]分割结果的影响
割方法 ${ }^{[23]}$ 、基于最小割优化的分割方法 ${ }^{[18]}$ 和基于 谱聚类的分割方法 ${ }^{[5]}$ 的结果. 上述方法的展示结果 均来自其对应文献. 人工分割结果由普林斯顿分 割数据集 ${ }^{[27]}$ 提供. 从图 11 可以看出, 上述分割方 法在人体模型的肘关节区域，由于分割特征较弱, 均没有进行分割, 而本文方法得到分割结果与人 工分割结果比较相似，完成了模型的语义分割.

本文测试环境为 $3.30 \mathrm{GHz}$ Intel Core i5-4590 CPU, 16GB 内存, 64 位 Windows 10 操作系统, 单 线程. 对于 MPI-FAUST 数据库 ${ }^{[26]}$, 每个人体模型 有 $1.3 \times 10^{4}$ 个三角面片，本文方法的平均运行时间 为 $7.249 \mathrm{~s}$, 其中骨架提取的时间为 $5.087 \mathrm{~s}$, Laplacian 矩阵特征分解的时间为 $1.409 \mathrm{~s}$. 对于 SCAPE 数据库 ${ }^{[25]}$, 每个人体模型有 $2.5 \times 10^{4}$ 个三角面片, 本文方法的平均运行时间为 $21.460 \mathrm{~s}$, 其中骨架提 取时间为 $14.031 \mathrm{~s}$, Laplacian 矩阵特征分解的时间 为 $5.769 \mathrm{~s}$.

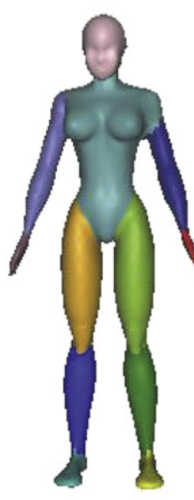

a. 文献[23]

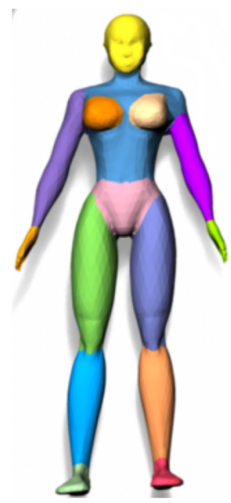

b. 文献[18]

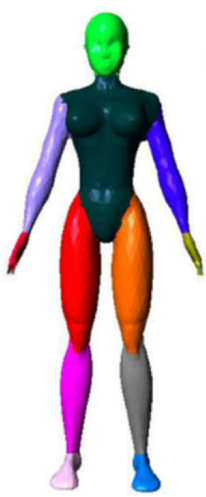

c. 文献[5]

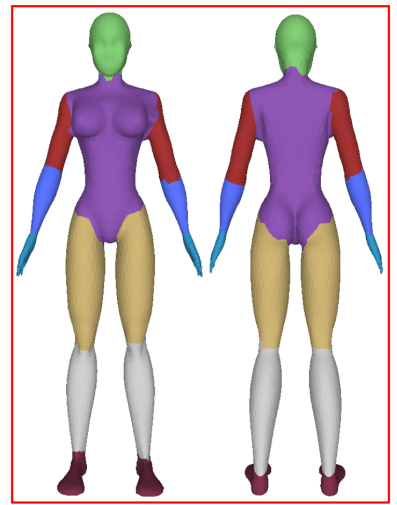

d. 人工分割 ${ }^{[27]}$

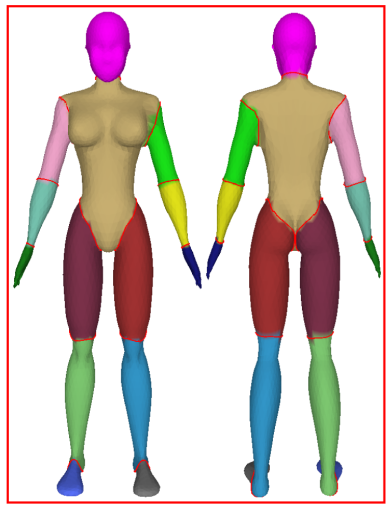

e. 本文

图 11 不同分割方法的可视化结果 2

本文方法依赖于模型的运动骨架，本文采用 的人体模型运动骨架提取方法 ${ }^{[4]}$ 适用于穿着少量 衣物的人体模型, 通过识别人体的固有属性获取 模型的语义信息. 普林斯顿分割数据集 ${ }^{[27]}$ 中部分 人体模型由于穿着宽大衣物或有较长头发导致模 型语义的缺失, 无法提取准确的运动骨架, 本文方 法不适用于这些模型. 未来将探索更鲁棒的骨架 提取方法，使本文方法的应用范围更广.

本文方法通过曲率将模型分成特征明显的区 域和不明显的区域．由于人体踝关节区域凹区域 不明显, 且模型曲率会随着人体姿态发展变化, 本 文方法在模型踝关节区域的分割线不完全一致, 未来将考虑对其进行进一步处理得到一致的分割 结果.

\section{7 结 语}

本文提出了一个自动的、对称可区分的三维人 体模型的语义分割方法, 不需要学习过程即可获 得模型分割块的语义. 该方法通过设置模板骨架 的分割标签, 自动确定模型分割块的数量, 避免了 模型过分割或欠分割的情况, 实现了分割块数量 可控的模型语义分割. 在生成模型分割线时, 本文 方法借助骨架自动确定了调和场的边界约束, 自 适应地选取取值区间生成分割线候选集; 采用最 小主曲率对模型分割特征明显的区域和分割特征 不明显的区域进行了区分，选取了不同的方式对 分割线进行篮选, 并以谱聚类的分割结果引导特 征明显区域分割线的篮选, 获取了其中准确的分 
割线. 实验结果表明, 本文方法能正确地生成模型 的左右可区分的语义分割结果，且对人体模型的 形状和姿态比较鲁棒.

由于人体模型对称区域的特征相似，基于学 习的方法通常不区分人体模型对称区域的语义信 息. 本文方法通过提取对称可区分的、带有语义信 息的运动骨架，并将其语义信息传递至模型分割 块, 得到了对称可区分的人体模型的语义分割结 果. 相对传统的模型分割方法来说, 本文方法能自 动获取分割块的数量, 避免了过分割和欠分割的 情况. 本文完成了三维人体模型的语义分割，下一 步将考虑将本文方法推广到其他模型，提高方法 的通用性。

\section{参考文献(References):}

[1] Hanocka R, Hertz A, Fish N, et al. MeshCNN: a network with an edge[J]. ACM Transactions on Graphics, 2019, 38(4): Article No.90

[2] Shu Z Y, Shen X Y, Xin S Q, et al. Scribble-based 3D shape segmentation via weakly-supervised learning[J]. IEEE Transactions on Visualization and Computer Graphics, 2020, 26(8): 2671-2682

[3] Shu Zhenyu, Yang Sipeng, Xin Shiqing, et al. 3D shape segmentation algorithm using weighted energy adaptive distribution[J]. Journal of Computer-Aided Design \& Computer Graphics, 2020, 32(3): 343-351(in Chinese)

(舒振宇，杨思鹏，辛士庆，等. 基于权重能量自适应分布的 三维形状分割算法 [J]. 计算机辅助设计与图形学学报, 2020, 32(3): 343-351)

[4] Luo S, Feng J Q. Symmetry-aware kinematic skeleton generation of a 3D human body model[J]. Multimedia Tools and Applications, 2020, 79(29/30): 20579-20602

[5] Zhang H Y, Wu C L, Deng J S, et al. A new two-stage mesh surface segmentation method[J]. The Visual Computer, 2018, 34(11): 1597-1615

[6] Zhang X, Li G Q, Xiong Y H, et al. 3D mesh segmentation using mean-shifted curvature[C] //Proceedings of International Conference on Geometric Modeling and Processing. Heidelberg: Springer, 2008: 465-474

[7] Fan L B, Liu L G, Liu K. Paint mesh cutting[J]. Computer Graphics Forum, 2011, 30(2): 603-612

[8] Benjamin W, Polk A W, Vishwanathan S V N, et al. Heat walk: robust salient segmentation of non-rigid shapes[J]. Computer Graphics Forum, 2011, 30(7): 2097-2106

[9] Chen L J, Georganas N D. An efficient and robust algorithm for 3D mesh segmentation[J]. Multimedia Tools and Applications, 2006, 29(2): 109-125

[10] van Kaick O, Fish N, Kleiman Y, et al. Shape segmentation by approximate convexity analysis[J]. ACM Transactions on Graphics, 2014, 34(1): Article No.4
[11] Zhang H J, Li C, Gao L L, et al. Shape segmentation by hierarchical splat clustering[J]. Computers \& Graphics, 2015, 51: 136-145

[12] Fang Y, Sun M T, Kim M, et al. Heat-mapping: a robust approach toward perceptually consistent mesh segmentation[C] //Proceedings of the IEEE Conference on Computer Vision and Pattern Recognition. Los Alamitos: IEEE Computer Society Press, 2011: 2145-2152

[13] Zhang J Y, Zheng J M, Wu C L, et al. Variational mesh decomposition[J]. ACM Transactions on Graphics, 2012, 31(3): Article No.21

[14] Chahhou M, Moumoun L, EI Far M, et al. Segmentation of 3D meshes using p-spectral clustering[J]. IEEE Transactions on Pattern Analysis and Machine Intelligence, 2014, 36(8): 1687-1693

[15] Hoffman D D, Singh M. Salience of visual parts[J]. Cognition, 1997, 63(1): 29-78

[16] Lee Y, Lee S, Shamir A, et al. Mesh scissoring with minima rule and part salience[J]. Computer Aided Geometric Design, 2005, 22(5): 444-465

[17] Golovinskiy A, Funkhouser T. Randomized cuts for 3D mesh analysis[J]. ACM Transactions on Graphics, 2008, 27(5): Article No.145

[18] Tong W H, Yang X K, Pan M D, et al. Spectral mesh segmentation via $\ell_{0}$ gradient minimization[J]. IEEE Transactions on Visualization and Computer Graphics, 2020, 26(4): 1807-1820

[19] Zheng Y Y, Tai C L. Mesh decomposition with cross-boundary brushes[J]. Computer Graphics Forum, 2010, 29(2): 527-535

[20] Meng M, Fan L B, Liu L G. iCutter: a direct cut-out tool for 3D shapes[J]. Computer Animation and Virtual Worlds, 2011, 22(4): 335-342

[21] Zheng Y Y, Tai C L, Au O K C. Dot scissor: a single-click interface for mesh segmentation[J]. IEEE Transactions on Visualization and Computer Graphics, 2012, 18(8): 1304-1312

[22] Au O K C, Zheng Y Y, Chen M L, et al. Mesh segmentation with concavity-aware fields[J]. IEEE Transactions on Visualization and Computer Graphics, 2012, 18(7): 1125-1134

[23] Wang H, Lu T, Au O K C, et al. Spectral 3D mesh segmentation with a novel single segmentation field[J]. Graphical Models, 2014, 76(5): 440-456

[24] Ji Z P, Liu L G, Chen Z G, et al. Easy mesh cutting[J]. Computer Graphics Forum, 2006, 25(3): 283-291

[25] Anguelov D, Srinivasan P, Koller D, et al. SCAPE: shape completion and animation of people[J]. ACM Transactions on Graphics, 2005, 24(3): 408-416

[26] Bogo F, Romero J, Loper M, et al. FAUST: dataset and evaluation for 3D mesh registration[C] //Proceedings of the IEEE Conference on Computer Vision and Pattern Recognition. Los Alamitos: IEEE Computer Society Press, 2014: 3794-3801

[27] Chen X B, Golovinskiy A, Funkhouser T. A benchmark for 3D mesh segmentation[J]. ACM Transactions on Graphics, 2009, 28(3): Article No.73

[28] Shapira L, Shamir A, Cohen-Or D. Consistent mesh partitioning and skeletonisation using the shape diameter function[J]. The Visual Computer, 2008, 24(4): 249-259 